\title{
Erratum to: Risk of psychiatric treatment for mood disorders and psychotic disorders among migrants and Dutch nationals in Utrecht, The Netherlands
}

\author{
J. P. Selten $\cdot$ W. Laan $\cdot$ R. Kupka $\cdot$ H. M. Smeets $\cdot$ \\ J. van Os
}

Published online: 24 September 2014

(c) Springer-Verlag Berlin Heidelberg 2014

Erratum to: Soc Psychiatry Psychiatr Epidemiol (2012)

47:271-278

DOI 10.1007/s00127-010-0335-7

In the original publication of this article, there were some errors in Tables 1 and 2. The corrected versions of the tables are shown here.

The online version of the original article can be found under doi:10.1007/s00127-010-0335-7.

J. P. Selten

Rivierduinen Institute for Mental Health Care, Oegstgeest,

The Netherlands

J. P. Selten $(\varangle)$

De School, Endegeesterstraatweg 2, 2342 AJ Oegstgeest,

The Netherlands

e-mail: j.selten@ggzleiden.nl

W. Laan · H. M. Smeets

Julius Center for Health Sciences and Primary Care,

University Medical Centre Utrecht, Utrecht, The Netherlands

R. Kupka

Altrecht Institute for Mental Health Care, Utrecht,

The Netherlands

J. P. Selten · J. van Os

Department of Psychiatry and Psychology, South Limburg

Mental Health Research and Teaching Network, EURON,

Maastricht University Medical Centre, Maastricht, The

Netherlands 
Table 1 Relative risks for ethnic minorities in the town of Utrecht, adjusted for age and sex, of treatment at a mental health institute for unipolar depressive disorder or bipolar disorder, 1 January 2002 to 31 December 2006

\begin{tabular}{|c|c|c|c|c|c|c|c|}
\hline \multirow[t]{2}{*}{ Ethnic group } & \multirow{2}{*}{$\begin{array}{l}\text { Person-years } \\
\mathrm{M} / \mathrm{F}\end{array}$} & \multicolumn{3}{|c|}{ Unipolar depressive disorder ${ }^{\mathrm{a}}$} & \multicolumn{3}{|c|}{ Bipolar disorder $^{\mathrm{b}}$} \\
\hline & & $\mathrm{M} / \mathrm{F}$ & $\mathrm{RR}$ & $(95 \% \mathrm{CI})$ & $\mathrm{M} / \mathrm{F}$ & RR & $(95 \% \mathrm{CI})$ \\
\hline \multicolumn{8}{|c|}{ First generation, $15-65$ years } \\
\hline Dutch nationals ${ }^{c}$ & $334,624 / 357,508$ & $563 / 976$ & 1.0 & Ref & $142 / 163$ & 1.0 & Ref \\
\hline Turkish & $15,669 / 14,887$ & $131 / 214$ & 4.6 & $(4.1-5.2)$ & $4 / 5$ & 0.6 & $(0.3-1.2)$ \\
\hline Moroccan & $31,270 / 27,621$ & $179 / 278$ & 3.6 & $(3.2-3.9)$ & $7 / 5$ & 0.5 & $(0.3-0.9)$ \\
\hline Surinamese & $8,628 / 9,310$ & $4 / 57$ & 1.7 & $(1.3-2.1)$ & $2 / 4$ & 0.6 & $(0.3-1.3)$ \\
\hline Western-European ${ }^{\mathrm{d}}$ & $6,364 / 6,852$ & $4 / 16$ & 0.7 & $(0.4-1.1)$ & $4 / 3$ & 1.4 & $(0.6-2.9)$ \\
\hline \multicolumn{8}{|c|}{ Second generation, $15-65$ years } \\
\hline Dutch nationals ${ }^{\mathrm{c}}$ & $334,624 / 357,508$ & $563 / 976$ & 1.0 & Ref & $142 / 163$ & 1.0 & Ref \\
\hline Turkish & $5,445 / 5,125$ & $16 / 48$ & 5.0 & $(3.8-6.5)$ & $1 / 3$ & 2.7 & $(1.0-7.3)$ \\
\hline Moroccan & 6,919/6,674 & $8 / 34$ & 2.7 & $(1.9-3.7)$ & $2 / 0$ & 1.1 & $(0.3-4.5)$ \\
\hline Surinamese & $4,160 / 4,247$ & $5 / 21$ & 2.1 & $(1.4-3.1)$ & $1 / 3$ & 2.3 & $(0.9-6.3)$ \\
\hline Western-European $^{\mathrm{e}}$ & $8,432 / 8,927$ & $15 / 27$ & 1.1 & $(0.8-1.4)$ & $4 / 3$ & 0.8 & $(0.4-1.7)$ \\
\hline \multicolumn{8}{|c|}{ First and second generation combined, $15-65$ years } \\
\hline Dutch nationals $^{\mathrm{c}}$ & $334,624 / 357,508$ & $563 / 976$ & 1.0 & Ref & $142 / 163$ & 1.0 & Ref \\
\hline Turkish & $21,114 / 20,112$ & $147 / 262$ & 4.9 & $(4.4-5.5)$ & $5 / 8$ & 0.8 & $(0.5-1.5)$ \\
\hline Moroccan & $38,189 / 34,295$ & $187 / 312$ & 3.6 & $(3.3-4.0)$ & $9 / 5$ & 0.5 & $(0.3-0.9)$ \\
\hline Surinamese & $12,788 / 13,557$ & $29 / 78$ & 1.8 & $(1.5-2.2)$ & $3 / 7$ & 0.8 & $(0.4-1.6)$ \\
\hline Western-European & $14,796 / 15,779$ & $19 / 43$ & 0.9 & $(0.7-1.2)$ & $8 / 6$ & 1.0 & $(0.6-1.7)$ \\
\hline
\end{tabular}

a The DSM-IV diagnoses major depressive disorder, single or recurrent episode (codes $296.2 \times$ or $296.3 \times$ )

b The DSM-IV diagnoses for bipolar I or II disorder or for bipolar disorder not otherwise specified (codes $296.0 \times, 296.4 \times, 296.5 \times, 296.6 \times$, 296.7, 296.89, 296.80)

${ }^{c}$ Born in the Netherlands and both parents born in the Netherlands

d Born in France, UK, Germany or Belgium

e Born in the Netherlands and one or both parents born in France, UK, Germany or Belgium. If parents were born in different countries, country of birth of mother determines ethnicity 
Table 2 Relative risks for ethnic minorities in the town of Utrecht, adjusted for age and sex, of treatment at a mental health institute for non-affective psychotic disorder, 1 January 2002 to 31 December 2006

\begin{tabular}{|c|c|c|c|}
\hline Ethnic group ${ }^{\mathrm{a}}$ & $\mathrm{M} / \mathrm{F}$ & $\mathrm{RR}$ & $(95 \% \mathrm{CI})$ \\
\hline \multicolumn{4}{|c|}{ First generation, $15-65$ years } \\
\hline Dutch nationals ${ }^{\mathrm{b}}$ & $364 / 283$ & 1.0 & Ref \\
\hline Turkish & $28 / 14$ & 1.3 & $(1.0-1.8)$ \\
\hline Moroccan & $106 / 22$ & 2.3 & $(1.9-2.8)$ \\
\hline Surinamese & $46 / 24$ & 3.4 & $(2.6-4.3)$ \\
\hline Western European ${ }^{\mathrm{c}}$ & $4 / 4$ & 0.7 & $(0.3-1.4)$ \\
\hline \multicolumn{4}{|c|}{ Second generation, $15-65$ years } \\
\hline Dutch nationals ${ }^{\mathrm{b}}$ & $364 / 283$ & 1.0 & Ref \\
\hline Turkish & $18 / 4$ & 3.6 & $(2.4-5.6)$ \\
\hline Moroccan & $17 / 4$ & 2.8 & $(1.8-4.3)$ \\
\hline Surinamese & $13 / 11$ & 4.3 & $(2.9-6.5)$ \\
\hline Western European ${ }^{\mathrm{d}}$ & $13 / 4$ & 0.9 & $(0.6-1.5)$ \\
\hline \multicolumn{4}{|c|}{ First and second generation combined, $15-65$ years } \\
\hline Dutch nationals $^{\mathrm{b}}$ & $364 / 283$ & 1.0 & Ref \\
\hline Turkish & $46 / 18$ & 1.8 & $(1.4-2.4)$ \\
\hline Moroccan & $123 / 26$ & 2.5 & $(2.1-3.0)$ \\
\hline Surinamese & $59 / 35$ & 3.8 & $(3.0-4.7)$ \\
\hline Western European & $17 / 8$ & 0.9 & $(0.6-1.3)$ \\
\hline
\end{tabular}

The DSM-IV diagnoses schizophrenia, schizophreniform disorder, schizoaffective disorder, delusional disorder, brief psychotic disorder or psychotic disorder not otherwise specified

${ }^{\text {a }}$ For person-years at risk: see Table 1

${ }^{\mathrm{b}}$ Born in the Netherlands and both parents born in the Netherlands

c Born in France, UK, Germany or Belgium

${ }^{\mathrm{d}}$ Born in the Netherlands and one or both parents born in France, UK, Germany or Belgium. If parents were born in different countries, country of birth of mother determines ethnicity 